\title{
一个强荧光三维超分子有机框架的构建及其对苦味酸的选择性传感
}

\author{
吴义鹏 ${ }^{a}$ 王泽坤 ${ }^{a} \quad$ 王辉 $^{a} \quad$ 张丹维 ${ }^{a} \quad$ 赵新*, $b$ 黎占亭*, $a$ \\ $\left({ }^{a}\right.$ 复旦大学化学系 上海市分子催化与功能材料重点实验室 上海 200438) \\ $\left({ }^{b}\right.$ 中国科学院上海有机化学研究所 有机功能分子合成与组装化学重点实验室 上海 200032)
}

\begin{abstract}
摘要 在四苯基甲烷外侧通过 $\mathrm{C}=\mathrm{C}$ 双键引入四个 $N$-甲基-4-苯基吡啶盐片段, 合成了一个新的全共轭单体 T-1. 其在水 中与 2 equiv.的葫芦腿[8]结合, 形成了新的高强荧光的超分子有机框架 SOF-r-SPP. 动态光散射实验表明, 在 T-1 浓度 为 1.0 和 $0.031 \mathrm{mmol} / \mathrm{L}$ 时, SOF-r-SPP 的流体力学直径分别为 68 和 $41 \mathrm{~nm}$. 粉末 X-射线衍射实验揭示, SOF-r-SPP 形成 孔径为 $2.3 \mathrm{~nm}$ 的三维金刚石型周期性结构. 硝基苯衍生物能有效淬灭 SOF-r-SPP 的苂光. 在 17 个研究的硝基苯类分 子中, 苦味酸的淬灭效率最高. SOF-r-SPP 作为苂光传感器, 对苦味酸的检测极限可以达到 $0.024 \mu \mathrm{mol} / \mathrm{L}$.
\end{abstract}

关键词＼cjkstart超分子有机框架; 菼光传感; 苦味酸; 葫芦[8]腿; 自组装

\section{Self-Assembly of a Highly Fluorescent Three-Dimensional Supramolecular Organic Framework and Selective Sensing for Picric Acid}

\author{
Wu, Yi-Peng ${ }^{a} \quad$ Wang, Ze-Kun ${ }^{a} \quad$ Wang, Hui $^{a} \quad$ Zhang, Dan-Wei $^{a} \quad$ Zhao, Xin ${ }^{*}, b$ \\ Li, Zhan-Ting*,a
}

$\left({ }^{a}\right.$ Department of Chemistry, Shanghai Key Laboratory of Molecular Catalysis and Innovative Materials, Fudan University, Shanghai 200438, China)

$\left({ }^{b}\right.$ Key Laboratory of Synthetic and Self-Assembly Chemistry for Organic Functional Molecules, Shanghai Institute of Organic Chemistry, Chinese Academy of Sciences, Shanghai 200032, China)

\begin{abstract}
Cucurbit[8]uril (CB[8])-encapsulation-based host-guest chemistry has been utilized to construct supramolecular organic frameworks, a family of water-soluble, self-assembled periodic porous structures, from multi-armed preorganized building blocks. The tetrahedral prototype building block has been incorporated with four $\mathrm{CH}_{2}$ units to connect the central tetraphenylmethane and appended aromatic arms. Herein we designed and prepared a new fully conjugated tetrahedral building block T-1, which possesses four $N$-methyl 4-(4-styrylphenyl)pyridinium (SPP) arms. The $1: 2$ mixture of T-1 with CB[8] in water leads to the formation of a new three-dimensional homogeneous diamondoid supramolecular organic framework SOF-r-SPP through CB[8] encapsulation for intermolecular dimers of the appended SPP units. ${ }^{1} \mathrm{H}$ NMR, absorption and fluorescence experiments conformed strong binding between the two components at diluted concentrations and $1: 2$ binding stoichiometry. Isothermal calorimetric (ITC) experiments established that the three-component $(\mathrm{SPP})_{2} \subset \mathrm{CB}[8]$ complexes formed between the SPP units of T-1 and $\mathrm{CB}[8]$ had an apparent binding constant of $5.5 \times 10^{13} \mathrm{M}^{-2}$, which was $5.5 \times 10^{4}$ times as high as that of the complex of a SPP control. ITC experiments also revealed that the self-assembly of SOF-r-SPP are driven both enthalpically and entropically, but the enthalpic contribution was overwhelmingly higher. Dynamic light scattering experiments revealed that within the concentration range of $0.031 \mathrm{mmol} / \mathrm{L}$ to $1.0 \mathrm{mmol} / \mathrm{L}$ of $\mathbf{T}-1$, the framework possessed a hydrodynamic diameter of $41 \mathrm{~nm}$ to $68 \mathrm{~nm}$. Molecular modelling study indicated that the new regular framework formed an aperture of $2.3 \mathrm{~nm}$. Although T-1 has nearly no fluorescence, SOF-r-SPP exhibits strong fluorescence in water probably due to the encapsulation of the SPP dimers by $\mathrm{CB}[8]$ that suppresses the relative rotation of the aromatic rings. Adding nitrobenzene or naphthalene derivatives to the solution of SOF-r-SPP remarkably quenched the fluorescence of the framework. Among other sixteen nitro-bearing aromatic molecules, picric acid (2,4,6-trinitrophenol) exhibited the largest quenching ability. At the low concentration of $1.0 \mu \mathrm{mol} / \mathrm{L}$ for T-1 of SOF-r-SPP, $0.1 \mu \mathrm{mol} / \mathrm{L}$ of 2,4,6-tirnitrophenol could cause $16 \%$ quenching of the fluorescence of SOF-r-SPP and $0.1 \mathrm{mmol} / \mathrm{L}$ of 2,4,6-tirnitrophenol could realize nearly complete quench $(>97 \%)$. Following a reported method, the limit of detection of SOF-r-SPP for picric acid was as low as 0.024 $\mu \mathrm{mol} / \mathrm{L}$.
\end{abstract}

Keywords supramolecular organic framework; fluorescence sensing; 2,4,6-trinitrophenol; cucurbituril; self-assembly

*E-mail: xzhao@sioc.ac.cn; ztli@fudan.edu.cn; Tel.: 021-31242038

Received June 15, 2019; published June 28, 2019.

Supporting information for this article is available free of charge via the Internet at http://sioc-journal.cn.

Project supported by the National Natural Science Foundation of China (Nos. 21432004, 21890732).

项目受国家自然科学基金(Nos. 21432004, 21890732)资助. 


\section{1 引言}

超分子聚合物是由分子单体通过可逆非共价键驱 动形成的一类动态聚合物结构 ${ }^{[114]}$. 基于多臂单体的超 分子聚合物具有二维和三维网络特征 ${ }^{[15 ~ 30]}$, 其分子间 作用也可以通过多价性协同作用获得增强 ${ }^{[31 ~ 35]}$, 与主 链型超分子聚合物相比具有更高的稳定性. 理论上由多 臂分子形成的超分子聚合物可以形成孔型网络结构. 但 当骨架为柔性结构时, 很难获得有序性的孔结构. 对金 属有机框架和共价有机框架的研究表明 ${ }^{[36 \sim 43]}$, 当有机 分子单体或连接子为刚性结构时, 可以通过离子静电作 用或配位作用及形成动态共价键, 获得高度稳定的固相 有序孔结构. 当以方向性的氢键为驱动力或以两个不同 单体间的配位作用为驱动力时, 也可以构建固相氢键有 机框架或超分子配位框架体系 ${ }^{[44]}$.

2013 年以来, 我们研究组与合作者利用葫芦腿 [8] (CB[8])在水中对疏水性芳香基团二聚体的包结增强效 应，构建了水溶性的超分子有机框架(Supramolecular Organic Frameworks, SOFs $)^{[25 ~ 29]}$. 利用这一策略, 其他 研究组也相继组装了不同形式的二维单层有序结 构 ${ }^{[20 ~ 23]}$. 我们进一步利用四苯基甲烷和三 $\left(2,2^{\prime}\right.$ - 联二吡 啶)钉络合物为节点, 构建了一系列四面体和八面体单 体, 其与 $\mathrm{CB}[8]$ 结合可以形成三维的金刚石型或立方型 SOF 结构 ${ }^{[45 \sim 53]}$, 并分别在药物吸收和输送及光诱导质 子还原产生氢气等方面获得应用 ${ }^{[47 \sim 52]}$. 为进一步拓展 $\mathrm{SOF}$ 的结构和功能, 需要在多臂单体中引入具有特定性 质的共轭基团. 基于这一设想, 我们提出把共轭基团 4-(4-苯乙烯苯基)吡啶盐(4-(4-styrylphenyl)pyridinium, SPP)引入到单体分子中, 合成了新的全刚性(rigid)单体 分子 T-1(图 1), 由此组装了一个新的高度稳定的超分子 有机框架 SOF-r-SPP, 并揭示了其对硝基苯类分子的高

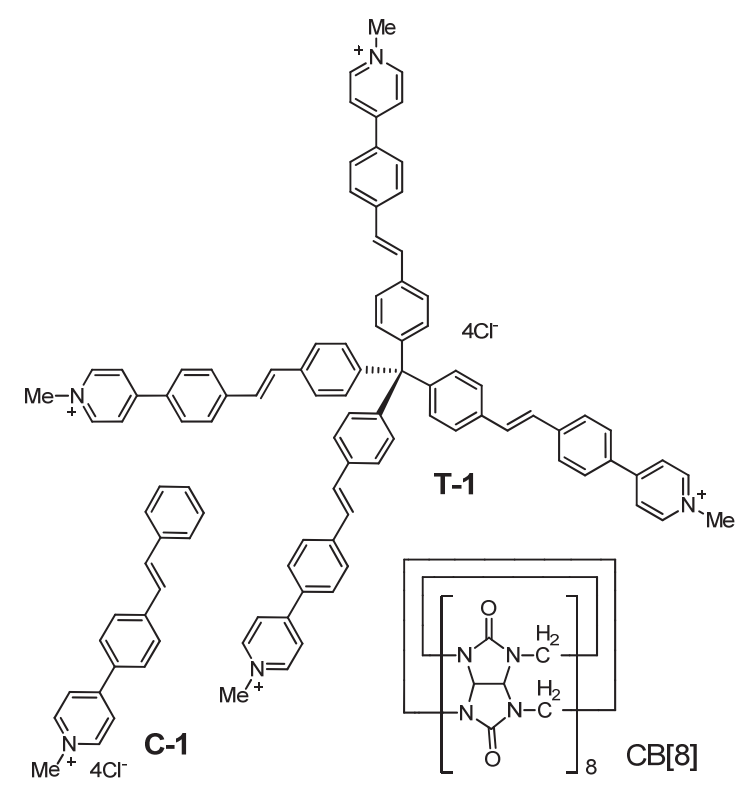

图 1 化合物 T-1, C-1 和 CB[8]的结构

Figure 1 The structures of compounds $\mathbf{T}-\mathbf{1}, \mathbf{C}-\mathbf{1}$ and $\mathrm{CB}[8]$
灵敏传感和对苦味酸 $(2,4,6$-三硝基苯酚)的高选择性和 高灵敏性传感. 本文报道详细结果.

\section{2 结果与讨论}

化合物 T-1 的合成路线见图 2. 四溴化合物 $\mathbf{1}^{[54]}$ 和亚 磷酸三乙酯在乙腈中加热回流反应 $12 \mathrm{~h}$, 生成四磷酸二 乙酯 $\mathbf{2}$, 产率为 $81 \%$. 以叔丁醇钾为碱, $\mathbf{2}$ 与化合物 $\mathbf{3}$ 反 应形成四吡啶衍生物 $\mathbf{4}$, 产率为 $75 \%$. 化合物 4 与碘甲 烷在乙腈中回流反应 $8 \mathrm{~h}$, 后经全氟磷酸铵和四丁基氯 化铵离子交换, 以 $84 \%$ 的产率生成四面体单体 T-1. 我 们进一步合成了控制化合物 C-1(图 1). 首先通过化合物 $\mathbf{3}^{[46]}$ 和 $\mathbf{5}^{[5]}$ 的反应以 $91 \%$ 产率制备出中间体 $\mathbf{6}$ (图 2), 其 与碘甲烷反应后，再经过两次离子交换，以 $84 \%$ 产率制 备出 C-1. 化合物 T-1 和 C-1 在水中具有较高的溶解性, 这是与水溶性很低的 $\mathrm{CB}[8]$ 在水中形成水溶性有序自组 装结构的前提. 由于 $\mathrm{Cl}^{-}$具有更高的溶剂化能力, 交换 为 $\mathrm{Cl}^{-}$可以显著提高 $\mathbf{T}-1$ 和 $\mathbf{C}-1$ 在水中的溶解度.

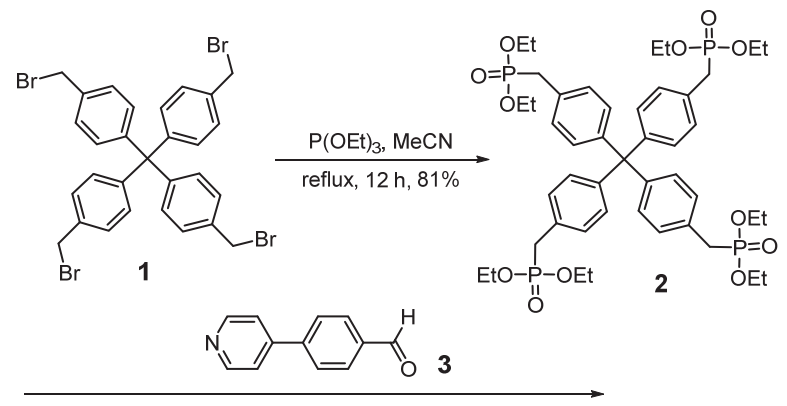

$t$-BuOK, $t$-BuOH/THF $(1: 10), 70{ }^{\circ} \mathrm{C}, 16 \mathrm{~h}, 75 \%$

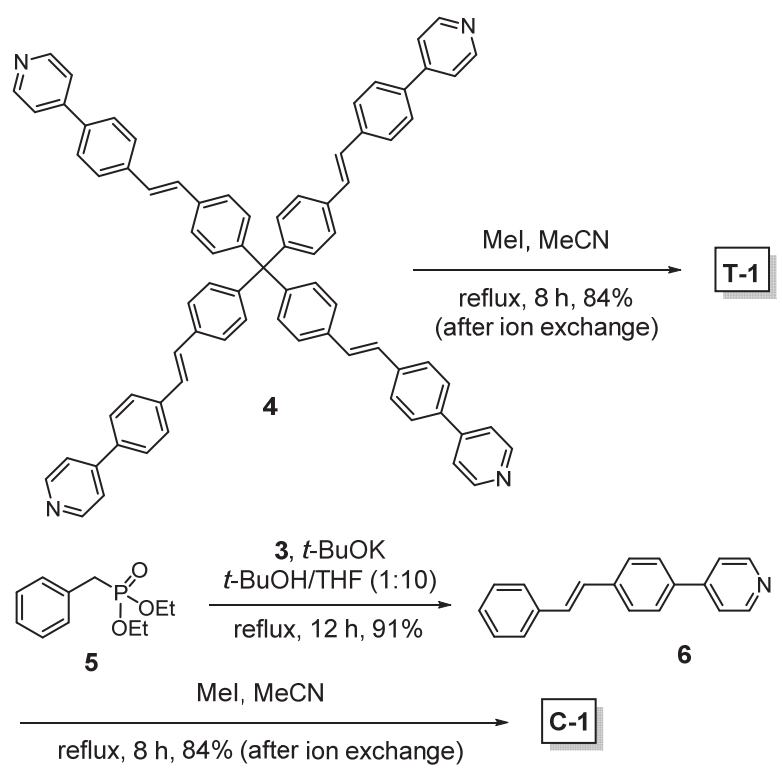

图 2 化合物 T-1 和 C-1 的合成路线

Figure 2 The synthesis of compounds T-1 and C-1

$\mathrm{CB}[8]$ 在水中溶解度很小 $(<0.01 \mathrm{mmol} / \mathrm{L})^{[56]}$. 在 $\mathrm{D}_{2} \mathrm{O}$ 中的 ${ }^{1} \mathrm{H}$ NMR 研究表明, 加入不同比例的 T-1 到 $\mathrm{CB}[8]$ 的 $\mathrm{D}_{2} \mathrm{O}$ 悬浮液中可以提高 $\mathrm{CB}[8]$ 的溶解度, 而 $\mathbf{T}-1$ 
的信号分辨率则大幅度降低, 表明二者形成了复杂的络 合物形式(支持信息图 S1). 把不同当量的化合物 C-1 加 入到 $\mathrm{CB}[8]$ 在 $\mathrm{D}_{2} \mathrm{O}$ 的悬浮液中也可以增溶 $\mathrm{CB}[8]$. 在 $\mathbf{C - 1}$ 达到相对于 $\mathrm{CB}[8]$ 当量的 $1 / 2$ 之前形成一套不同于游离 的 C-1 的信号(支持信息图 S2), 表明二者主要形成了 1: 1 包结络合物. 增加 C-1 的量导致两套信号产生. 在 加到 2 equiv. 时, 形成另一套分辨率较高的信号, 表明 二者形成了 $1: 2$ 包结络合物 ${ }^{[57,58]}$, 也间接支持了 $\mathbf{T}-\mathbf{1}$ 与 $\mathrm{CB}[8]$ 的最佳结合计量比为 $1: 2$.

在 T-1 的水溶液中逐渐加入 $\mathrm{CB}[8]$ 导致了其长波长 区域吸收峰明显降低, 并产生红移, 最大红移 $29 \mathrm{~nm}$ (图 $3)$. 在加入 2 equiv. CB[8]后, 吸光度不再进一步降低, 并且最大吸收波长也不再红移. 这一结果表明, 二者以 $1: 2$ 计量比实现最佳结合. 在 $\mathbf{C}-1$ 的水溶液中加入 $\mathrm{CB}[8]$ 也导致前者的吸收光谱变弱和红移现象(支持信 息图 S3). 尽管在加入 0.5 equiv. $\mathrm{CB}[8]$ 时, 吸光度降低 有一个比较显著的拐点(此时红移约 $15 \mathrm{~nm}$ ), 进一步加 入 $\mathrm{CB}[8]$ 仍能够产生较弱的吸光度降低, 表明过量的 $\mathrm{CB}[8]$ 可能诱导 $1: 2$ 的包结络合物转化为 $1: 1$ 的包结 络合物. 上述结果显示, T-1 的外侧苯基吡啶与 $\mathrm{CB}[8]$ 形 成的 $2: 1$ 络合物更稳定. 基于荧光发射光谱的 Job 图也 证明, 二者的结合计量比为 $1: 2$ (支持信息图 S4). 等温 量热滴定实验揭示, $\mathrm{CB}[8]$ 与 $\mathbf{T - 1}$ 的 SPP 基团形成的 1 : 2 包结络合物的表观结合常数为 $5.5( \pm 1.7) \times 10^{13} \mathrm{M}^{-2}$, 而 $\mathrm{CB}[8]$ 与 $\mathbf{C - 1}$ 形成的 $1: 2$ 络合物的结合常数为 $1.0( \pm$ $0.11) \times 10^{9} \mathrm{M}^{-2}$. 前者高于后者 $5.5 \times 10^{4}$ 倍, 表明 T-1 与 $\mathrm{CB}[8]$ 的结合具有显著的多价性(支持信息图 S5). T-1 的 SPP 形成的络合物的 $\Delta H$ 和 $-T \Delta S$ 分别为 $-13.1 \mathrm{kcal}$ 和 $-1.6 \mathrm{kcal}$, 表明二者的结合是焓和熵同时驱动的过程, 而前者占支配地位.

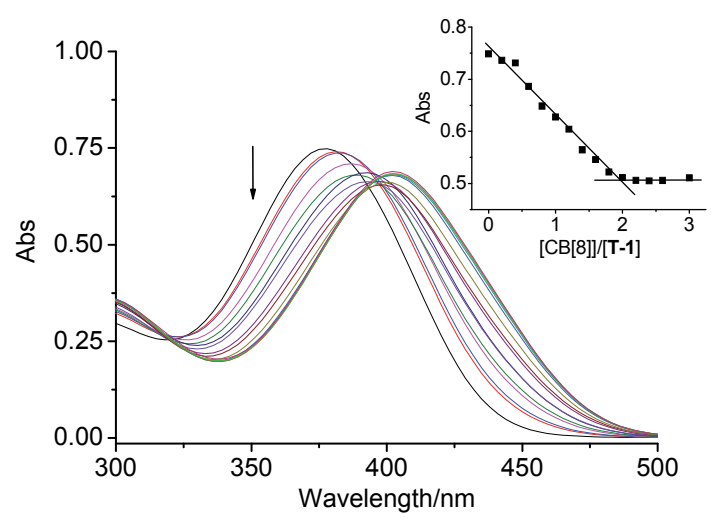

图 3 化合物 T-1 的水溶液 $(12.5 \mu \mathrm{mol} / \mathrm{L})$ 在不同当量 $\mathrm{CB}[8]$ 存在下的吸 收光谱. 内嵌图为 T-1 的在 $374 \mathrm{~nm}$ 处吸光度与二者当量比的关系图 Figure 3 The absorption spectra of compound T-1 $(12.5 \mu \mathrm{mol} / \mathrm{L})$ in water at $25{ }^{\circ} \mathrm{C}$ in the presence of incremental amount of $\mathrm{CB}[8]$. Inset: the absorbance of $\mathbf{T}-\mathbf{1}$ at $374 \mathrm{~nm}$ versus $[\mathrm{CB}[8]] /[\mathbf{T}-\mathbf{1}]$

动态光散射实验揭示, 在 $1.0 \mathrm{mmol} / \mathrm{L}$ 浓度时, T-1 在 水中形成流体力学直径 $\left(D_{\mathrm{H}}\right)$ 为 $4 \mathrm{~nm}$ 的组装体, 表明这
一四面体单体在水中发生了一定程度的簇集. 而同样浓 度的 T-1 与 $\mathrm{CB}[8]$ (2 mmol/L) 在水中能形成大的组装体, $D_{\mathrm{H}}$ 达到 $68 \mathrm{~nm}$ (图 4). 浓度降低导致 $D_{\mathrm{H}}$ 变小. 但当浓度 降低至 $0.031 \mathrm{mmol} / \mathrm{L}$ 时, $D_{\mathrm{H}}$ 仍能达到 $41 \mathrm{~nm}$, 显示出这 一自组装结构的高稳定性. 旋转流变实验揭示, 在室温 下四面体单体 T-1 (1.0 mmol/L) 在水中的剪切粘度为 8.0 $\times 10^{-3} \mathrm{~Pa} \cdot \mathrm{s}$. 与之对比的是, 相同浓度的 T-1 与 2 equiv. 的 CB[8]形成的组装体剪切粘度增加到 $0.30 \mathrm{~Pa} \cdot \mathrm{s}$. 所有 这些实验表明，二者形成了较大的自组装结构 SOF-r-SPP (图 5).

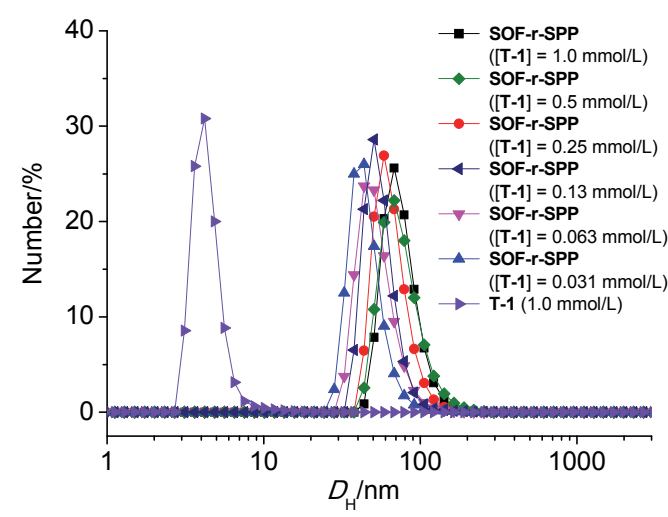

图 4 动态光散射实验测得的化合物 T-1 $(1.0 \mathrm{mmol} / \mathrm{L})$ 和其与 $\mathrm{CB}[8]$ 的 $1: 2$ 的水溶液中形成的组装体 SOF-r-SPP 的流体力学直径

Figure 4 The hydrodynamic diameter of T-1 $(1.0 \mathrm{mmol} / \mathrm{L})$ and its $1: 2$ mixture with $\mathrm{CB}[8]$ in water obtained using dynamic light scattering experiments at $25{ }^{\circ} \mathrm{C}$

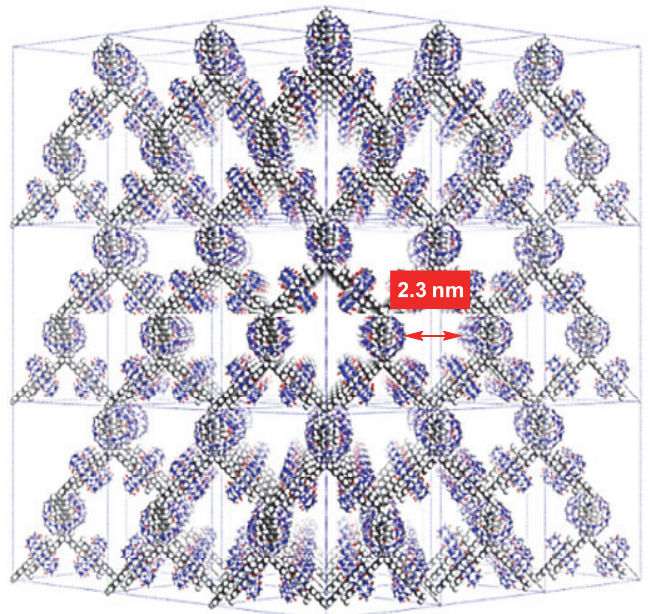

图 5 超分子有机框架 MOF-r-SPP 的模拟结构(由 Materials Studio 7.0 软件模拟 $)^{[44]}$, 其孔径为 $2.3 \mathrm{~nm}$

Figure 5 Model of 3D supramolecular organic framework MOF-r-SPP formed by $\mathbf{T}-1$ and $\mathrm{CB}[8](1: 2)$, which has a pore aperture of $2.3 \mathrm{~nm}$. The structure was obtained by Materials Studio $7.0^{[44]}$

室温缓慢挥发 $1: 2$ 的 T-1 和 CB[8]的水溶液得到二 者形成的玻璃状固体组装体. 粉末 X-射线衍射(PXRD) 实验揭示了两个较宽但也较强的衍射峰，分别位于 $2 \theta$ $=6.2^{\circ}$ 和 $10.2^{\circ}$ 处 (图 6a). 这两个信号分别对应于模拟的 三维结构的 $\{222\}$ 和 $\{600\}$ 晶面, 晶面间距分别为 1.5 和 
$0.8 \mathrm{~nm}$. 在同步辐射 X-射线线站获得的图谱中 ${ }^{[59,60]}$, 这 一样品展示出三个衍射峰 $\left(2 \theta=2.4^{\circ}, 5.1^{\circ}\right.$ 和 $\left.8.4^{\circ}\right)$, 分别 与模拟计算的 $\{111\},\{222\}$ 和 $\{600\}$ 晶面匹配，对应于 $3.0,1.4$ 和 $0.8 \mathrm{~nm}$ 的晶面间距(图 6b). 两个实验都支持二 者形成了新的周期性的超分子有机框架 SOF-f-SPP(图 5).
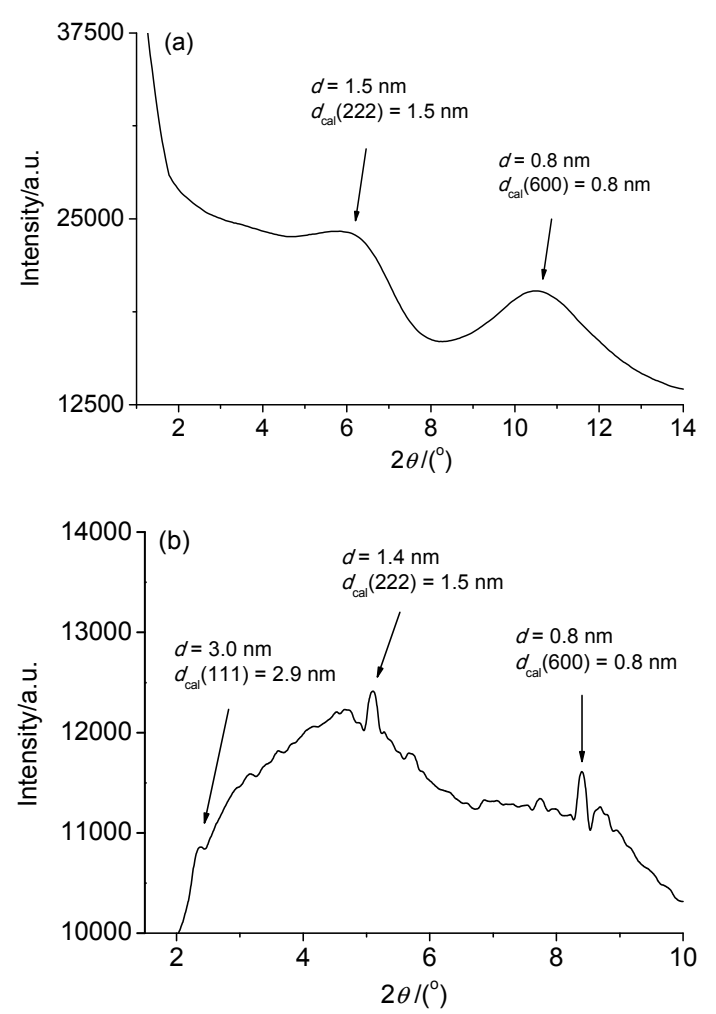

图 6 SOF-r-SPP 的(a) PXRD 光谱和(b)同步辐射 PXRD 光谱

Figure 6 (a) PXRD profile and (b) synchrotron radiation PRXD profile of solid-state SOF-r-SPP

场发射透射电镜揭示, SOF-r-SPP 在固态呈现规整 的微晶形貌。微晶固体选区电子衍射实验也证明了 $\{111\}$ 和 $\{220\}$ 晶面的存在, 晶面间距分别为 2.9 和 1.8 $\mathrm{nm}$ (支持信息图 S6), 与上述 X-射线衍射结果相一致. 能 量色散 X-射线光谱研究进一步揭示了这一微晶的元素 的清晰分布(支持信息图 S7), C, N, O 和 $\mathrm{Cl}$ 元素占比分别 为 $72.3 \%, 17.4 \%, 9.9 \%$ 和 $0.4 \%$, 与理论计算值吻合.

四面体单体 T-1 在水中的荧光非常弱, 而同样 T-1 浓度的 SOF-r-SPP 则产生非常强的荧光(图 7). 可能的 原因是, $\mathrm{CB}$ [8]包结的 SPP 二聚体被隔离了溶剂水分子, 并且吡啶和连结的苯环的相对旋转也受到抑制, 从而产 生类似聚集诱导增强发光的效应 ${ }^{[61,62]}$. 加入硝基苯类分 子 G1 G17(图 8) 可以显著淬灭 SOF-r-SPP 的苂光. 加 入 $0.1 \mathrm{mmol} / \mathrm{L}$ 的 $\mathbf{G 2} \sim \mathbf{G 1 7}$ 到 $\mathbf{T}-1$ 为 $1.0 \mu \mathrm{mol} / \mathrm{L}$ 的 SOF-r-SPP 中, 导致后者荧光降低 $21 \% \sim 55 \%$. 而同样 浓度的苦味酸 $\mathbf{G 1}$ 则几乎全部淬灭其苂光 $(>97 \%)$, 显示 出高度的选择性(图 7, 内嵌图). 苂光滴定实验揭示, 加 入 G1 到 SOF-r-SPP 的水溶液中导致苂光持续降低(支
持信息图 S8). $0.1 \mu \mathrm{mol} / \mathrm{L}$ 的 G1 即能导致 SOF-r-SPP 的 $16 \%$ 的苂光淬灭. 参照已经确立的方法处理苂光淬灭光 谱 $^{[63]}$, 揭示对 $\mathbf{G 1}$ 的检测极限在 $0.024 \mu \mathrm{mol} / \mathrm{L}$. 这一高灵 敏性可能反映了框架结构的有序结构固定了 4-苯基吡 啶盐二聚体与中间的四(乙烯基苯基)甲烷间的距离, G1 作为缺电性受体与较为富电性的四(乙烯基苯基)甲烷间 产生最强的受体一供体相互作用，从而导致最有效的荧 光淬灭.

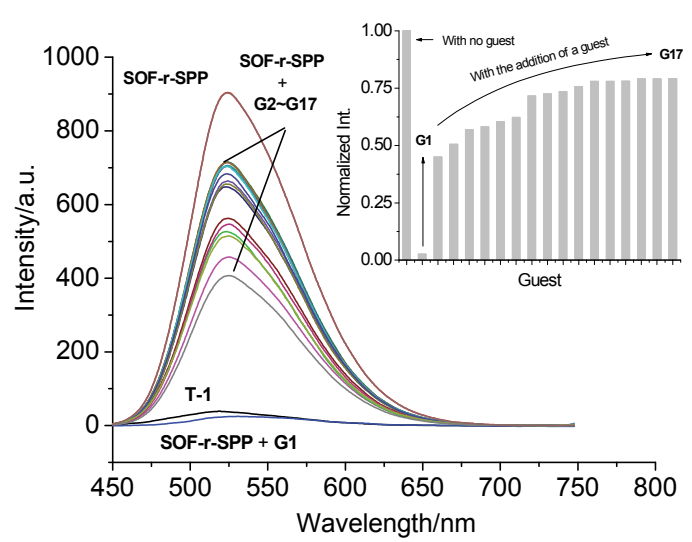

图 $7 \quad$ T-1 $(1.0 \mu \mathrm{mol} / \mathrm{L})$ 和加入客体 $\mathbf{G 1} \sim \mathbf{G 1 7}(0.1 \mathrm{mmol} / \mathrm{L})$ 的 SOF-r-SPP $([\mathbf{T}-1]=1.0 \mu \mathrm{mol} / \mathrm{L})$ 水溶液的荧光光谱图 $\left(\lambda_{\mathrm{ex}}=380 \mathrm{~nm}\right)$. 内 嵌图: 在硝基苯类客体 $\mathbf{G 1} \sim \mathbf{G 1 7}(0.1 \mathrm{mmol} / \mathrm{L})$ 存在下的 SOF-r-SPP $([\mathrm{T}-1]=1.0 \mu \mathrm{mol} / \mathrm{L})$ 的水溶液的荧光发射峰的归一化(相对于不加客体 的 SOF-r-SPP 溶液的苂光强度)后的相对强度

Figure 7 The fluorescent spectra of T-1 $(1.0 \mu \mathrm{mol} / \mathrm{L})$ and SOF-r-SPP $([\mathbf{T}-\mathbf{1}]=1.0 \mu \mathrm{mol} / \mathrm{L})$ after the addition of guests $\mathbf{G 1} \sim \mathbf{G 1 7}(0.1 \mathrm{mmol} / \mathrm{L})$ $\left(\lambda_{\mathrm{ex}}=380 \mathrm{~nm}\right.$ ). Inset: normalized (relative to that of SOF-r-SPP in the absence of a guest) fluorescence intensity of SOF-r-SPP $([\mathbf{T}-\mathbf{1}]=1.0$ $\mu \mathrm{mol} / \mathrm{L})$ in water in the presence of nitrobenzene guests $\mathbf{G 1} \sim \mathbf{G 1 7}(0.1$ $\mathrm{mmol} / \mathrm{L}$ )<smiles>O=[N+]([O-])c1cc([N+](=O)[O-])c(O)c([N+](=O)[O-])c1</smiles><smiles>Cc1ccc([N+](=O)[O-])c(O)c1</smiles><smiles>O=[N+]([O-])c1cccc(O)c1</smiles><smiles>Nc1ccc([N+](=O)[O-])cc1</smiles><smiles>O=[N+]([O-])c1cccc2ccccc12</smiles><smiles>O=[N+]([O-])c1ccc(O)cc1[N+](=O)[O-]</smiles><smiles>O=Cc1ccc([N+](=O)[O-])cc1</smiles><smiles>O=[N+]([O-])c1cc(CO)cc([N+](=O)[O-])c1</smiles>

G5<smiles>O=C(O)c1ccc([N+](=O)[O-])cc1</smiles>

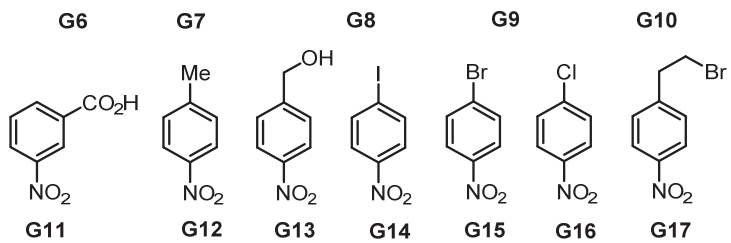

图 8 化合物 $\mathbf{G 1} \sim \mathbf{G 1 7}$ 的结构

Figure 8 The structure of compounds $\mathbf{G 1} \sim \mathbf{G 1 7}$

上述用于苂光测试的稀溶液浓缩到 $[\mathbf{T}-1]=0.1$ $\mathrm{mmol} / \mathrm{L}$ 后, 用氯仿萃取去除硝基苯类客体. 动态光散 射实验表明, 溶液中仍能形成 $D_{\mathrm{H}}$ 约为 $40 \mathrm{~nm}$ 的组装体, 表明硝基苯类客体不破坏框架结构. 


\section{3 结论}

我们设计合成了一个新的全共轭四面体单体, 其与 $\mathrm{CB}[8]$ 在水中组装形成一个具有高荧光的超分子有机框 架 SOF-r-SPP. 新的框架结构对苦味酸具有高灵敏度的 检测能力 ${ }^{[64 ~ 66]}$, 检测极限浓度达到 $10^{-8} \mu \mathrm{mol} / \mathrm{L}$. 具有 特定尺度空穴的框架结构研究近年来受到广泛重视. 基 于主客体结合原理形成的超分子有机框架具有独特的 水溶性特点. SOF-r-SPP 作为苂光传感材料的高灵敏性 预示, 空穴尺度更大的均相框架结构有可能用于感应生 物大分子特别是活性蛋白. 我们未来将设计新的高水溶 性的连接臂更长的单体分子, 以探索超分子有机框架在 此方面的应用潜力.

\section{4 实验部分}

化合物 2. 将化合物 $\mathbf{1}^{[54]}(0.70 \mathrm{~g}, 1.0 \mathrm{mmol})$ 和亚磷 酸三乙酯 $(0.85 \mathrm{~g}, 5.00 \mathrm{mmol})$ 溶于乙腈 $(25 \mathrm{~mL})$ 中, 回流 反应 $12 \mathrm{~h}$ 后冷却至室温. 用旋转蒸发仪除去溶剂后, 向 剩余物中加入正己烷 $(100 \mathrm{~mL})$. 反复振荡后过滤产生的 沉淀, 沉淀再用正己烷和乙醚 $(1: 1, V / V)$ 洗涤 3 次, 然 后用正己烷和乙酸乙酯 $(10: 1, V / V)$ 重结晶, 得到化合 物 2 为浅白色固体 $(0.75 \mathrm{~g}, 81 \%)$, 熔点: $305{ }^{\circ} \mathrm{C}$ (分解). ${ }^{1} \mathrm{H}$ NMR (400 MHz, Chloroform- $d$ ) $\delta: 7.22 \sim 7.11(\mathrm{~m}, 2 \mathrm{H})$, $7.07(\mathrm{~d}, J=8.1 \mathrm{~Hz}, 2 \mathrm{H}), 3.98\left(\mathrm{~d}, \mathrm{q}, J_{1}=8.1 \mathrm{~Hz}, J_{2}=3.7\right.$ $\mathrm{Hz}, 4 \mathrm{H}), 3.10(\mathrm{~d}, J=21.6 \mathrm{~Hz}, 2 \mathrm{H}), 1.88 \sim 0.52(\mathrm{~m}, 6 \mathrm{H})$. ${ }^{13} \mathrm{C}$ NMR (101 MHz, Chloroform- $d$ ) $\delta$ : 145.1, 131.1, 129.3, 128.9, 62.2, 33.8, 32.5, 16.5. ${ }^{31} \mathrm{P}$ NMR (162 MHz, Chloroform- $d$ ) $\delta$ : 26.5. HRMS (ESI): Calcd for $\mathrm{C}_{45} \mathrm{H}_{64} \mathrm{O}_{12} \mathrm{P}_{4}: 920.3348\left[\mathrm{M}+\mathrm{NH}_{4}\right]^{+}$. Found: 938.3681.

化合物 4. 把化合物 $2(0.15 \mathrm{~g}, 0.16 \mathrm{mmol})$ 和 $\mathbf{3}^{[67]}$ $(0.18 \mathrm{~g}, 1.00 \mathrm{mmol})$ 加入到叔丁醇和四氢呋喃混合溶剂 $(1: 10, V / V, 10 \mathrm{~mL})$ 中, 然后一次性加入叔丁醇钾 $(0.22$ $\mathrm{g}, 2.0 \mathrm{mmol})$. 在 $70{ }^{\circ} \mathrm{C}$ 下搅拌反应 $16 \mathrm{~h}$ 后冷却至室温. 用旋转蒸发仪除去溶剂后向混合物中加入水 $(20 \mathrm{~mL})$. 反复振荡后过滤形成的沉淀, 用甲醇 $(5 \mathrm{~mL} \times 3)$ 和二氯 甲烷 $(5 \mathrm{~mL} \times 3)$ 洗涤沉淀后, 用 $N, N$-二甲基甲酰胺重结 晶, 得化合物 4 为黄色固体 $(0.12 \mathrm{~g}, 75 \%)$. 熔点 $>$ $300{ }^{\circ} \mathrm{C}$ (分解). ${ }^{1} \mathrm{H}$ NMR $\left(400 \mathrm{MHz}, \mathrm{CF}_{3} \mathrm{CO}_{2} \mathrm{H}\right) \delta: 8.72(\mathrm{~d}$, $J=5.0 \mathrm{~Hz}, 8 \mathrm{H}), 8.33(\mathrm{~d}, J=6.2 \mathrm{~Hz}, 8 \mathrm{H}), 7.91(\mathrm{~d}, J=7.9$ $\mathrm{Hz}, 8 \mathrm{H}), 7.80(\mathrm{~d}, J=7.9 \mathrm{~Hz}, 8 \mathrm{H}), 7.56(\mathrm{~d}, J=7.8 \mathrm{~Hz}, 8 \mathrm{H})$, $7.49 \sim 7.34(\mathrm{~m}, 12 \mathrm{H}), 7.23(\mathrm{~d}, J=16.1 \mathrm{~Hz}, 4 \mathrm{H}) \cdot{ }^{13} \mathrm{C}$ NMR $\left(100 \mathrm{MHz}, \mathrm{CF}_{3} \mathrm{CO}_{2} \mathrm{H}\right) \delta: 159.8,147.1,143.0,140.4,134.5$, $131.9,131.7,131.4,128.0,127.6,126.3,126.0,123.6$. HRMS (ESI): Calcd for $\mathrm{C}_{77} \mathrm{H}_{56} \mathrm{~N}_{4}: 1036.4505[\mathrm{M}+\mathrm{H}]^{+}$. Found: 1037.4578 .

化合物 T-1. 将化合物 $4(0.12 \mathrm{~g}, 0.11 \mathrm{mmol})$ 加入到 乙腈 $(10 \mathrm{~mL})$ 中, 然后加入碘甲烷 $(0.18 \mathrm{~g}, 1.28 \mathrm{mmol})$. 加 热回流 $8 \mathrm{~h}$ 后冷却至室温, 将其中深黄色固体过滤后溶 于最少量的水中. 向该溶液中滴加饱和六氟磷酸铵水溶
液, 直至不再形成更多沉淀. 过滤后所得沉淀用冷水洗 涤, 然后溶解在最少量乙腈中. 向该溶液中滴加饱和的 四丁基氯化铵水溶液, 再将产生的沉淀过滤并用冷乙腈 洗涤, 真空干燥后得到化合物 T-1 为浅黄色固体 $(0.11 \mathrm{~g}$, $84 \%$ ). 熔点 $>300{ }^{\circ} \mathrm{C}$ (分解). ${ }^{1} \mathrm{H}$ NMR $(400 \mathrm{MHz}$, DMSO- $\left.d_{6}\right) \delta: 8.98(\mathrm{~d}, J=6.4 \mathrm{~Hz}, 8 \mathrm{H}), 8.52(\mathrm{~d}, J=6.4 \mathrm{~Hz}$, $8 \mathrm{H}), 8.12(\mathrm{~d}, J=8.0 \mathrm{~Hz}, 8 \mathrm{H}), 7.85(\mathrm{~d}, J=8.0 \mathrm{~Hz}, 8 \mathrm{H}), 7.65$ (d, $J=8.4 \mathrm{~Hz}, 8 \mathrm{H}), 7.49$ (s, 4H), 7.38 (s, 4H), 7.29 (d, $J=$ $8.2 \mathrm{~Hz}, 8 \mathrm{H}), 4.31$ (s, $12 \mathrm{H}) .{ }^{13} \mathrm{C}$ NMR $(101 \mathrm{MHz}$, DMSO- $\left.d_{6}\right) \delta: 158.7,151.4,150.7,146.1,139.8,137.3$, 136.0, 133.7, 132.7, 132.6, 131.7, 128.7, 52.2. HRMS (ESI): Calcd for: $\mathrm{C}_{81} \mathrm{H}_{68} \mathrm{~N}_{4}: 274.1356[\mathrm{M}-4 \mathrm{Cl}]^{4+}$. Found: 274.1349.

化合物 6. 把苄澳 $(1.3 \mathrm{~g}, 8.0 \mathrm{mmol})$ 和亚磷酸三乙酯 $(1.7 \mathrm{~g}, 10.0 \mathrm{mmol})$ 加入乙腈 $(50 \mathrm{~mL})$ 中, 回流反应 $12 \mathrm{~h}$ 后 冷至室温, 用旋转蒸发仪除去溶剂, 再向混合物中加入 正己烷 $(200 \mathrm{~mL})$, 反复振荡后倾倒出上层溶剂. 重复上 述操作三次, 油状产物逐渐变为固体, 过滤形成的沉淀, 并用正己烷洗涤固体, 再真空干燥. 将得到的固体粗产 品 5 和 $3(1.8 \mathrm{~g}, 10 \mathrm{mmol})$ 溶解在叔丁醇和四氢呋喃的混 合溶剂中 $(1: 10, V / V, 50 \mathrm{~mL})$, 随后加入固体叔丁醇钾 $(2.2 \mathrm{~g}, 2.0 \mathrm{mmol})$. 加热回流 $12 \mathrm{~h}$ 后冷却至室温, 向混合 物中加入水 $(200 \mathrm{~mL})$, 过滤形成的沉淀, 用水 $(50 \mathrm{~mL} \times$ $3)$ 和丙酮 $(50 \mathrm{~mL} \times 3)$ 洗涤沉淀, 然后真空干燥, 再在乙 腈和 DMF $(5: 1, V / V)$ 中重结晶, 过滤干燥后得化合物 6 为白色固体 $(1.8 \mathrm{~g}, 91 \%)$, 熔点: $162{ }^{\circ} \mathrm{C} .{ }^{1} \mathrm{H}$ NMR $(400$ $\left.\mathrm{MHz}, \mathrm{CF}_{3} \mathrm{CO}_{2} \mathrm{H}\right) \delta: 8.65(\mathrm{~d}, J=5.1 \mathrm{~Hz}, 2 \mathrm{H}), 8.24(\mathrm{~d}, J=$ $5.0 \mathrm{~Hz}, 2 \mathrm{H}), 7.85(\mathrm{~d}, J=7.5 \mathrm{~Hz}, 2 \mathrm{H}), 7.73(\mathrm{~d}, J=7.3 \mathrm{~Hz}$, $2 \mathrm{H}), 7.51(\mathrm{~d}, J=7.3 \mathrm{~Hz}, 2 \mathrm{H}), 7.34(\mathrm{t}, J=7.1 \mathrm{~Hz}, 2 \mathrm{H})$, $7.30 \sim 7.23(\mathrm{~m}, 2 \mathrm{H}), 7.14(\mathrm{~d}, J=16.3 \mathrm{~Hz}, 1 \mathrm{H}) .{ }^{13} \mathrm{C}$ NMR $\left(101 \mathrm{MHz}, \mathrm{CF}_{3} \mathrm{CO}_{2} \mathrm{H}\right) \delta: 159.6,142.8,140.2,136.2,132.2$, $131.4,128.3,128.2,127.7,127.4,126.3,125.9,123.4$. Calcd for: $\mathrm{C}_{19} \mathrm{H}_{15} \mathrm{~N}: 257.1204[\mathrm{M}+\mathrm{H}]^{+}$. Found: 258.1292.

化合物 C-1. 将化合物 $6(0.18 \mathrm{~g}, 0.7 \mathrm{mmol})$ 悬浮在 乙腈 $(5 \mathrm{~mL})$ 中. 加入硒甲烷 $(0.14 \mathrm{~g}, 1.0 \mathrm{mmol})$ 后加热回 流 $8 \mathrm{~h}$, 冷却至室温. 沉淀过滤后干燥得粗产物为黄色 固体. 将粗产物溶于最少量水中, 再向其中滴加饱和六 氟磷酸铵水溶液, 直至没有沉淀产生. 过滤得沉淀物, 用冷水洗涤后再溶解在最少量乙腈中. 向该溶液中滴加 饱和四丁基氯化铵水溶液至没有沉淀产生. 将形成的沉 淀过滤，冷乙腈洗涤后真空干燥，得化合物 C-1 为淡黄 色固体 $(0.11 \mathrm{~g}, 84 \%)$. 熔点: $180{ }^{\circ} \mathrm{C}$ (分解). ${ }^{1} \mathrm{H}$ NMR $(400$ MHz, DMSO- $\left.d_{6}\right) \delta: 8.98(\mathrm{~d}, J=6.4 \mathrm{~Hz}, 2 \mathrm{H}), 8.53$ (d, $J=$ $6.4 \mathrm{~Hz}, 2 \mathrm{H}), 8.13(\mathrm{~d}, J=8.0 \mathrm{~Hz}, 2 \mathrm{H}), 7.87(\mathrm{~d}, J=8.0 \mathrm{~Hz}$, $2 \mathrm{H}), 7.66(\mathrm{~d}, J=7.6 \mathrm{~Hz}, 2 \mathrm{H}), 7.53(\mathrm{~s}, 1 \mathrm{H}), 7.49(\mathrm{~s}, 1 \mathrm{H})$, $7.46 \sim 7.23(\mathrm{~m}, 4 \mathrm{H}), 4.31(\mathrm{~s}, 3 \mathrm{H}) .{ }^{13} \mathrm{C}$ NMR $(101 \mathrm{MHz}$, DMSO- $\left.d_{6}\right) \delta: 154.0,145.9,141.4,137.0,132.5,131.7$, $129.3,128.9,128.8,128.0,127.5,127.3,124.0,47.4$. HRMS (ESI): Calcd for: $\mathrm{C}_{20} \mathrm{H}_{18} \mathrm{~N}: 272.1434[\mathrm{M}-\mathrm{Cl}]^{+}$. 
Found: 272.1453.

SOF-r-SPP 制备. 将 T-1 分子 $(25 \mathrm{mg}, 0.02 \mathrm{mmol})$ 溶 解于 $20 \mathrm{~mL}$ 蒸馏水中, 再加入 2.0 equiv. 的葫芦 [8]脲. 反 复超声加热，使葫芦[8]嫝完全溶解，即制得 SOF-r-SPP 溶液.

\section{致谢}

感谢上海光源 $(\mathrm{SSRF})$ 提供同步辐射 $\mathrm{X}$-射线衍射线 站(BL14B1)实验服务.

\section{References}

[1] Lehn, J.-M. Polym. Int. 2002, 51, 825.

[2] Brunsveld, L.; Folmer, B. J. B.; Meijer, E. W.; Sijbesma, R. P. Chem. Rev. 2001, 101, 4071.

[3] Xu, J.-F.; Zhang, X. Acta Polym. Sin. 2017, 37 (in Chinese). (徐江 飞, 张希, 高分子学报, 2017, 37.)

[4] Wang, Q.; Cheng, M.; Jiang, J.-L.; Wang, L.-Y. Chin. Chem. Lett. 2017, 28, 793 .

[5] Ding, Z.; Li, H.; Gao, W.; Zhang, Y.; Liu, C.; Zhu, Y. Chin. J. Chem. 2017, 35, 447.

[6] Zhao, Q.; Chen, Y.; Liu, Y. Chin. Chem. Lett. 2018, $29,84$.

[7] Wang, J.; Zou, Q.; Yan, X. Acta Chim. Sinica 2017, 75, 933 (in Chinese). (王娟, 邹千里, 间学海, 化学学报, 2017, 75, 933.)

[8] Zheng, X.; Miao, Q.; Wang, W.; Qu, D.-H. Chin. Chem. Lett. 2018, $29,1621$.

[9] Ji, S.; Xu, H. Sci. China Chem. 2019, 62, 155.

[10] Wang, X.; Yang, Y.; Fan, L.; Yang, F.; Wu, D. Sci. China Chem. 2018, 61, 311.

[11] Xu, C.; Xu, L.; Ma, X. Chin. Chem. Lett. 2018, 29, 970.

[12] Yin, G.; Chen, L.; Wang, C.; Yang, H. Chin. J. Chem. 2018, 36, 134.

[13] Chen, Y.; Sun, S.; Lu, D.; Shi, Y.; Yao, Y. Chin. Chem. Lett. 2019, $30,37$.

[14] Ma, L.; Peng, H.; Lu, X.; Liu, L.; Shao, X. Chin. J. Chem. 2018, 36, 845 .

[15] Albertazzi, L.; Martinez-Veracoechea, F. J.; Leenders, C. M. A.; Voets, I. K.; Meijer, E. W. Proc. Natl. Acad. Sci. U. S. A. 2013, 110, 12203.

[16] Fang, R.; Liu, Y.; Wang, Z.; Zhang, X. Polym. Chem. 2013, 4, 900.

[17] Xiao, X.; Sun, J.; Jiang, J. Chem. Eur. J. 2013, 19, 16891.

[18] Liu, Y.; Huang, Z.; Liu, K.; Kelgtermans, H.; Dehaen, W.; Wang, Z.; Zhang, X. Polym. Chem. 2014, 5, 53.

[19] Li, C.; Han, K.; Li, J.; Zhang, Y.; Chen, W.; Yu, Y.; Jia, X. Chem. Eur. J. 2013, 19, 11892.

[20] Pfeffermann, M.; Dong, R.; Graf, R.; Zajaczkowski, W.; Gorelik, T.; Pisula, W.; Narita, A.; Muellen, K.; Feng, X. J. Am. Chem. Soc. 2015, 137, 14525.

[21] Li, Y.; Dong, Y.; Miao, X.; Ren, Y.; Zhang, B.; Wang, P.; Yu, Y.; Li, B.; Isaacs, L.; Cao, L. Angew. Chem. Int. Ed. 2018, 57, 729.

[22] Lee, H.-J.; Kim, H.-J.; Lee, E.-C.; Kim, J.; Park, S. Y. Chem. Asian J. 2018, 13, 390 .

[23] Liu, H.; Zhang, Z.; Zhao, Y.; Zhou, Y.; Xue, B.; Han, Y.; Wang, Y.; Mu, X.; Zang, S.; Zhou, X.; Li, Z. J. Mater. Chem. B 2019, 7, 1435.

[24] Lin, Q.; Fan, Y.-Q.; Mao, P.-P.; Liu, L.; Liu, J.; Zhang, Y.-M.; Yao, H.; Wei, T.-B. Chem. Eur. J. 2018, 24, 777.

[25] Zhang, K.-D.; Tian, J.; Hanifi, D.; Zhang, Y.; Sue, A. C.-H.; Zhou, T.-Y.; Zhang, L.; Zhao, X.; Liu, Y.; Li, Z.-T. J. Am. Chem. Soc. 2013, $135,17913$.

[26] Xu, S.-Q.; Zhang, X.; Nie, C.-B.; Pang, Z.-F.; Xu, X.-N.; Zhao, X. Chem. Commun. 2015, 51, 16417.

[27] Wang, H.; Zhang, D.-W.; Zhao, X.; Li, Z.-T. Acta Chim. Sinica 2015, 73, 471 (in Chinese). (王辉, 张丹维, 赵新, 黎占亭, 化学学 报, 2015, 73, 471.)

[28] Tian, J.; Chen, L.; Zhang, D.-W.; Liu, Y.; Li, Z.-T. Chem. Commun. 2016, 52,6351

[29] Tian, J.; Wang, H.; Zhang, D.-W.; Liu, Y.; Li, Z.-T. Natl. Sci. Rev. 2017, 4, 426 .
[30] Zhao, Y.-K.; Gao, Z.-Z.; Wang, H.; Zhang, D.-W.; Li, Z.-T. Chin. Chem. Lett. 2019, 30, 127.

[31] Mulder, A.; Huskens, J.; Reinhoudt, D. N. Org. Biomol. Chem. 2004, 2,3409 .

[32] Badjic, J. D.; Nelson, A.; Cantrill, S. J.; Turnbull, W. B.; Stoddart, J. F. Acc. Chem. Res. 2005, 38, 723.

[33] Fasting, C.; Schalley, C. A.; Weber, M.; Seitz, O.; Hecht, S.; Koksch, B.; Dernedde, J.; Graf, C.; Knapp, E.-W.; Haag, R. Angew. Chem. Int. Ed. 2012, 51, 10472.

[34] Pieters, R. J. Org. Biomol. Chem. 2009, 7, 2013.

[35] Mahon, E.; Barboiu, M. Org. Biomol. Chem. 2015, 13, 10590.

[36] Cao, L.; Wang, T.; Wang, C. Chin. J. Chem. 2018, 36, 754.

[37] Zhang, H.; Li, G.; Zhang, K.; Liao, C. Acta Chim. Sinica 2017, 75, 841 (in Chinese). (张贺, 李国良, 张可刚, 廖春阳, 化学学报, 2017, 75, 841.)

[38] Yang, T.; Cui, Y.; Chen, H.; Li, W. Acta Chim. Sinica 2017, 75, 339 (in Chinese). (杨涛, 崔亚男, 陈怀银, 李伟华, 化学学报, 2017, 75, 339.)

[39] Wang, Y.; Yang, Q.; Su, B. Acta Chim. Sinica 2017, 75, 1071 (in Chinese). (王亚锋, 杨倩, 苏彬, 化学学报, 2017, 75, 1071.)

[40] Liang, R.-R.; Zhao, X. Org. Chem. Front. 2018, 5, 3341.

[41] Yuan, F.; Tan, J.; Guo, J. Sci. China Chem. 2018, 61, 143.

[42] Sun, B.; Wang, D.; Wan, L. Sci. China Chem. 2017, 60, 1098.

[43] Wu, M.-X.; Yang, Y.-W. Chin. Chem. Lett. 2017, 28, 1135.

[44] Lin, R.-B.; He, Y.; Li, P.; Wang, H.; Zhou, W.; Chen, B. Chem. Soc. Rev. 2019, 48, 1362.

[45] Tian, J.; Zhou, T.-Y.; Zhang, S.-C.; Aloni, S.; Altoe, M. V.; Xie, S.-H.; Wang, H.; Zhang, D.-W.; Zhao, X.; Liu, Y.; Li, Z.-T. Nat. Commun. 2014, 5, 5574.

[46] Tian, J.; Yao, C.; Yang, W.-L.; Zhang, L.; Zhang, D.-W.; Wang, H.; Zhang, F.; Liu, Y.; Li, Z.-T. Chin. Chem. Lett. 2017, 28, 798.

[47] Yao, C.; Tian, J.; Wang, H.; Zhang, D.-W.; Liu, Y.; Zhang, F.; Li, Z.-T. Chin. Chem. Lett. 2017, 28, 893.

[48] Wu, Y.-P.; Yang, B.; Tian, J.; Yu, S.-B.; Wang, H.; Zhang, D.-W.; Liu, Y.; Li, Z.-T. Chem. Commun. 2017, 53, 13367.

[49] Yu, S.-B.; Qi, Q.; Yang, B.; Wang, H.; Zhang, D.-W.; Liu, Y.; Li, Z.-T. Small 2018, 14, 1801037.

[50] Yan, M.; Liu, X.-B.; Gao, Z.-Z.; Wu, Y.-P.; Hou, J.-L.; Wang, H.; Zhang, D.-W.; Liu, Y.; Li, Z.-T. Org. Chem. Front. 2019, 6, 1698.

[51] Tian, J.; Xu, Z.-Y.; Zhang, D.-W.; Wang, H.; Xie, S.-H.; Xu, D.-W.; Ren, Y.-H.; Wang, H.; Liu, Y.; Li, Z.-T. Nat. Commun. 2016, 7, 11580.

[52] Li, X.-F.; Yu, S.-B.; Yang, B.; Tian, J.; Wang, H.; Zhang, D.-W.; Liu, Y.; Li, Z.-T. Sci. China Chem. 2018, 61, 830.

[53] Chen, Y.; Huang, F.; Li, Z.-T.; Liu, Y. Sci. China Chem. 2018, 61, 979.

[54] Tian, J.; Ding, Y.-D.; Zhou, T.-Y.; Zhang, K.-D.; Zhao, X.; Wang, H.; Zhang, D.-W.; Liu, Y.; Li, Z.-T. Chem. Eur. J. 2014, 20, 575.

[55] Wagner, S.; Accorsi, M.; Rademann, J. Chem. Eur. J. 2017, 23, 15387.

[56] Lee, J. W.; Samal, S.; Selvapalam, N.; Kim, H.-J.; Kim, K. Acc. Chem. Res. 2003, 36, 621.

[57] Zhang, Y.; Zhou, T.-Y.; Zhang, K.-D.; Dai, J.-L.; Zhu, Y.-Y.; Zhao, X. Chem. Asian J. 2014, 9, 1530.

[58] Yang, B.; Yu, S.-B.; Wang, H.; Zhang, D.-W.; Li, Z.-T. Chem. Asian J. 2018, 13, 1312 .

[59] Yang, T. Y.; Wen, W.; Yin, G. Z.; Li, X. L.; Gao, M.; Gu, Y. L.; Li, L.; Liu, Y.; Lin, H.; Zhang, X. M.; Zhao, B.; Liu, T. K.; Yang, Y. G.; Li, Z.; Zhou, X. T.; Gao, X. Y. Nucl. Sci. Tech. 2015, 26, 020101.

[60] Zeng, J.; Bian, F.; Wang, J.; Li, X.; Wang, Y.; Tian, F.; Zhou, P. J. Synchrotron Rad. 2017, 24, 509.

[61] He, Z.; Ke, C.; Tang, B. Z. ACS Omega 2018, 3, 3267.

[62] Qin, A.; Tang, B. Z. Sci. China Chem. 2018, 61, 879.

[63] Zhang, Y.; Zhan, T.-G.; Zhou, T.-Y.; Qi, Q.-Y.; Xu, X.-N.; Zhao, X Chem. Commun. 2016, 52, 7588.

[64] Yu, J.; Chen, Y.; Li, J.-J.; Liu, Y. J. Mater. Chem. C 2017, 5, 799.

[65] Wu, H.; Chen, Y.; Dai, X.; Li, P.; Stoddart, J. F.; Liu, Y. J. Am. Chem. Soc. 2019, 141, 6583.

[66] Zhang, Y.; Shen, P.; He, B.; Luo, W.; Zhao, Z.; Tang, B. Z. Polym. Chem. 2018, 9, 558 .

[67] Xiong, Z.; Wang, N.; Dai, M.; Li, A.; Chen, J.; Yang, Z. Org. Lett. 2004, 6, 3337.

(Cheng, B.) 